\title{
SOME CLINICAL PHASES OF OCULAR INVOLVEMENT IN SINUS DISEASE.
}

\author{
Edgar S. Thomson, M.D., new york City.
}

This subject is reviewed especially with reference to purulent sinuitis, eye lesions in which sinus disease cannot be demonstrated and functional disturbances of the optic nerve. It is illustrated by brief records of five cases. Read before the Academy of Medicine of Northern New Jersey, March 14th, 1921.

The influence on the eyes of sinus disease has received more or less attention in special literature for about twenty-five years or more. Numerous papers have been written and there is a rather decided unanimity of opinion on certain conditions but a diversity on others. As the question is to a great extent a matter of clinical judgment it may not be amiss to discuss it in the light of an individual experience.

Mackay, ${ }^{1}$ classifies the diseases of the nasal sinuses affecting the eye in four groups:

First; Mucoceles of the sinuses;

Second; Acute or chronic sinuitis with external signs of orbital cellulitis, orbital abscess, tumor growth, edema of the eyelids or apparent dacryocystitis;

Third; Sinuitis without external signs of orbital inflammation but with ophthalmoscopic signs such as optic neuritis, neuroretinitis, retinal thrombosis or phlebitis, or without ophthalmoscopic signs but with visual disturbances such as central scotoma, visual field defects, ocular muscle paralyses or fifth nerve disturbances;

Fourth; Cases in which the association of sinus disease has been asserted but which Mackay considers questionable, such as glaucoma, iritis, uveitis, keratitis, and lens and vitreous opacities. "Another class which is very important to the ophthalmologist has been omitted, namely the unilateral headaches and asthenopic symptoms due to rarefaction of the air within the frontal sinus and anterior ethmoidal cells." [Brawley. ${ }^{2}$ ]

It is perfectly evident as we glance over this classification that it covers a very wide field and is orderly only in so far as it relates to the clinical picture. It seems preferable, therefore, to speak of the sinus conditions and the effects they may produce more in accordance with what we know of the pathologic conditions.

\section{PURULENT SINUITIS.}

There seems no reason to doubt the existence of a certain type of case as of sinus causation, in view of the numerous cases which have been observed and reported. A sinus toxemia, of antral, frontal, ethmoidal or sphenoidal origin is perfectly capable of causing plastic inflammation of the iris, ciliary body, choroid or optic nerve. The uveal disease in such cases is sharp and severe in its type, and if an iritis, may be accompanied by hypopyon.

General symptoms as headache, fever, anemia and leucocytosis will usually be found, and the X-rays will show an increased density in the area of the affected sinus. Rhinologic diagnosis is only difficult if the purulent collection is shut off in a completely enciosed cell, as it may be in posterior ethmoidal and sphenoidal conditions. However, repeated nasal examinations with cautious exploration will often uncover the telltale drops of pus.

It is usual in such cases to have a rapid improvement in the eye condition following a radical sinus operation, an improvement which is practically confirmatory of the etiology, altho the complete subsidence of the eye disease may be a matter of some days if the iris be involved and of some weeks in the case of the choroid. Cases of this type presuppose a process of some duration and in such, a diagnosis is often not a difficult matter.

Purulent disease of the ethmoids or sphenoids may attack the eye, not so much by a general toxemia as by a more localized process. Iritis, choroiditis, cyclitis, or optic neuritis may occur, and the diagnosis may present considerable difficulty. In general it may be said that in the absence of local symptoms in the nose, or of any suggestive appearances in the X-ray examination, dependence must 
be placed in the absence of other causes for focal or general infection, in the teeth, blood, tonsils, or intestines, the type of the local process, and the fact that it is unilateral, as so many of these cases are apt to be.

Certain types of uveal disease are suggestive, a plastic iritis with or without hypopyon, (hypopyon is more apt to occur in a more extensive sinus process with general toxemia) a simple cyclitis with marked plastic deposits; choroiditis with marked plastic exudations in the choroid and retina or in the vitreous, or an optic neuritis with marked inflammatory exudates. In this connection it is - well to lay stress upon the difference between an optic neuritis with one or two diopters of swelling and marked plastic exudates and perhaps hemorrhages, and a choked disc with swelling of three or more diopters with slight inflammatory signs in proportion. If seen at the outset, the differential diagnosis should not be difficult. It is certainly very doubtful that sinus disease can produce a choked disc unless accompanied by cerebral complications which give rise to increased intracranial pressure.

A unilateral inflammation, iritis, cyclitis, choroiditis, or neuritis, is very suggestive of a local causation. This is particularly the case if the inflammation is of marked degree in one eye, and of some days' duration, while the other eye remains normal. If the causative agent be a general toxemia there seems on the face of it no reason why a susceptible tissue should be affected in one eye and not in the other. Clinical experience certainly bears this out; and it is unusual to find an iritis, for example, due to general toxemia in which both eyes are not affected altho one may be somewhat in advance of the other in point of time. These facts are well known but seem to be somewhat too frequently lost sight of, and have an important bearing in the diagnosis of sinus disease.

\section{EYE LESIONS IN WHICH SINUS DISEASE CANNOT BE DEMONSTRATED.}

There are two especial varieties of eye disease belonging to this class: First; Choroiditis, Second; Functional disturbances of the optic nerve.
Choroiditis is manifested by numerous fine "dusty" opacities all thru the vitreous. An indistinct view of the fundus can usually be obtained, but no choroidal exudates can be seen. It is very unlikely that any of these opacities are derived from the ciliary body as such cases do not primarily show changes in the intraocular tension and the ciliary anastomosis is not engorged. In fact, the vitreous opacities are the beginning and end of the clinical picture, and even after the process clears up, no atrophic spots can be found in the choroid. The vision is often surprisingly good but the patient complains of seeing numerous floating spots or "clouds." Flashes of light are not seen, or are very slight, certainly not so prominent a symptom as in a more extensive retinochoroiditis.

Later in the process the fellow eye may be involved thru extension of the process to the other side of the sinuses. Secondary glaucoma may supervene and is apt to be of the subacute type with rise of tension of 40 or $50 \mathrm{~mm}$. (Schiötz) and without much pain or redness; in other words, a type due to posterior retention of the lymphatic fluids rather than anterior. All search for a constitutional toxin is negative and the $\mathrm{X}$ rays give no suggestive findings.

Under such circumstances the ethmoids, particularly the posterior, and the sphenoid, must be well opened and good drainage established. To the rhinologist it seems as if he were operating on normal tissues. No pus nor exudation is found and no evidence of sinus disease. Soon after the operation, however, the ocular condition shows signs of improvement. No more opacities in the vitreous are formed, those present begin gradually to $a b$ sorb, and if increased tension be present it gradually comes down to normal. It is hardly necessary to say that complete resolution is a matter of some weeks or even months and that frequently a few fine opacities remain, but good vision should be the rule if the operation has not been too long delayed. Iridectomy is seldom required unless the process has been a long continued one with a long con- 
tinued elevation of tension. It is, moreover, very disappointing in its results and if it be done primarily the tension is likely to recur until the choroidal process is checked by the sinus operation. The seat of trouble is usually the ethmoids, probably the posterior ethmoids. The exact nature of the pathologic process is at present not definitely known.

Functional disturbances of the optic nerve. The most striking and characteristic type of this class of cases manifests itself by a partial central scotoma of about $20^{\circ}$ radius (concentric); with color blindness in the scotoma, and not the slightest appearance of optic nerve disease discoverable by the ophthalmoscope. The symptoms come on rapidly and may remain unchanged for a long period of time. The vision falls off to $20 / 50$ or $20 / 70$ and at times the test types show distortion. There are no general symptoms. $\mathrm{X}$-ray and rhinologic examinations are negative. The condition is always one sided, and has not the same tendency to involve the fellow eye that occurs in a purulent sinuitis. After a period of slight fuctuation the vision at times becomes steadily worse and entire perception of light may be lost. Of course, total atrophy of the optic nerve follows if the trouble is not promptly relieved at this stage.

The diagnosis is easily made in the most characteristic form of the disease, and yet general tests, Wassermann, teeth, tonsils, intestines, etc., should not be omitted. If the case be seen when the vision is very far gone the diagnosis must rest on the absence of fundus signs, the absence of visual disturbances in the fellow eye and the absence of other causation.

A frequent criticism has been made that such cases are hysteric and that they are relieved by the moral effect of the operation. There is no way of absolutely disproving this assertion until the true pathologic condition can be demonstrated. A very important point is as to whether the direct pupillary reaction corresponds with the amount of vision. It is usual for such cases to show a certain fluctuation in the vision and to this fluctuation the pupillary reaction should consistently correspond. Inconsistency is one of the most definite signs of an hysteric process. If the case be carefully studied an error in diagnosis should not often be made. It is certainly better to open the sinuses in a hysteric case than to allow a patient to acquire an optic atrophy from sinus disease in the presence of a wrong diagnosis of hysteric blindness.

As in the previous class of case, no evidence of sinus disease can be found during the operation. Some observers speak of finding the mucous membrane lining the sinuses, "dark, red and congested," but there seems to be no unanimity on this point, at least, among the rhinologists with whom I am acquainted. A free opening of the sphenoid and adjacent ethmoid cells should be made and in twenty-four to forty-eight hours the vision in the affected eye should begin to improve. Scotomas with only moderate decrease in vision may disappear entirely, while the more severe cases may show material gain, tho the final vision may not be secured for several weeks. In certain cases where perception of light is lost, the periphery of the field is first restored, the case passing thru the color scotoma phase, to finally normal vision. Where the case has gone on to atrophic changes in the optic nerve, it is usual to secure a marked improvement in vision if the operation has not been too long delayed.

As to the pathology of these cases, it seems on the face of it that the disturbance is most probably a mechanical one. Brawley" says that, "In some cases the process is undoubtedly a toxemia and the highly organized optic nerve fibres to the fovea are the first to suffer, which explains the frequency of central scotoma." It is true that the choroiditis cases mentioned above show enough exudation to suggest a toxemia, but the optic nerve cases, particularly the last mentioned class, recover too quickly to be explained in this manner. All demonstrably toxic lesions of the optic nerve, without exception, are slow to recover and restor- 
ation of vision is a matter of days or even weeks. Unquestionably the bleeding and depletion of the sinuses has a favorable influence, but it seems as if there must be some low grade inflammatory process, in the bone or periosteum which causes a fluid pressure on the nerve which subsides rapidly af. ter the operation, allowing a speedy restoration of function.

Finally the practical question confronts us: Are we to allow a dangerous process to go on merely because we cannot invariably demonstrate it with certainty? Opening the ethmoids and sphenoid is not a dangerous operation when done with proper care, and it is certainly better in doubtful cases to operate than to risk allowing a latent trouble to continue.

\section{CASES}

A few illustrative cases may be briefly cited. 1. L. Male, 44, first presented himself June 4, 1917. The right eye was slightly congested and he had a number of precipitates on the posterior surface of the cornea and a discolored iris. The attack had lasted several weeks, without much change. R. $V .=20 / 30+$ with +.50 cy. ax. $90^{\circ}$. He had had a similar, less severe, attack one year before which had recovered very slowly. General examination elicited little of importance. Xrays of the teeth showed no signs of a toxic process. His hemoglobin was $82 \%$. He had a moderate leucocytosis. He was somewhat pale and had a certain amount of intestinal putrefaction. Nasal examination by Dr. J. E. MacKenty showed nothing. X-ray examination of the sinuses showed no marked density changes.

$\mathrm{He}$ was treated with atropin and hot applications and finally, early in July, pus was found in the region of the sphenoid, by Dr. J. E. MacKenty. The ethmoids and sphenoid were opened and a radical operation performed by Dr. MacKenty, who found a purulent sinuitis of low grade.

The corneal precipitates rapidly absorbed and in ten days no traces of the ocular process could be discovered. There has been some difficulty in this case in keeping the sinus open as the bony tissue proliferates rapidly. If the sinus drainage becomes defective, a few floating spots appear in the vitreous or a few corneal precipitates appear. Proper drainage of the sinuses is at once followed by a clearing up of the ocular symptoms. His vision is now 20/15 in each eye.

Case 2. Mrs. S. 60, presented herself fortreatment May 7,1920. Aniridectomy for glaucoma had been done five weeks previously in the right eye and a week later in the left. Both eyes were red and inflamed, the left slightly worse. T. R. 23, L. 31 (Schiötz). R. V. = $20 / 40$; L. $V .=20 / 100$. The vitreous in each eye was full of fine dusty opacities, the left being worse than the right. Fields were normal. Wassermann negative, teeth negative by $\mathrm{X}$-rays, general examination showed only a slight heart murmur with good compensation. X-rays showed some slight bony changes in the region of the sphenoid. Nasal examination by Dr. J. E. MacKenty showed that the left ethmoids were not well open.

She was treated with pilocarpin and dionin, and vaccines were made from a streptococcus which was found by culture taken from the ethmoids. She was also given iodid of potassium. The ocular conditions remained unchanged and on June 11 th the tonometer readings were $\mathrm{R}$. 25 , L. 35. June 14 th, Dr. MacKenty opened the ethmoids" and sphenoid and found nothing. The following day the congestion of the eyeballs had markedly decreased and on June 21st, R. V. $=15 / 15-$; L. V. $15 / 20 \mathrm{~W}-.50$ cy. ax. $90^{\circ}$. Ton. Rt. 22. L. 25 . July 13th, the vitreous opacities had gone and tension was normal, which it has remained ever since. The vision is now R. 20/15 - with $0.25=-0.50 \mathrm{cy}$. ax. $75^{\circ}$. L. $20 / 20-$ with $=0.50 \frown-1.25 \mathrm{cy}$. ax. $75^{\circ}$. A few lens opacities remain. T, R. 20, L. 22.

Case. 3. C. Male, 19. While taking college examinations noticed that aspothad appeared in the left eye and that the letters appeared distorted. Two days later, Jan, 14, 1921, he presented himself for treatment. R. V. $=20 / 15$ with 


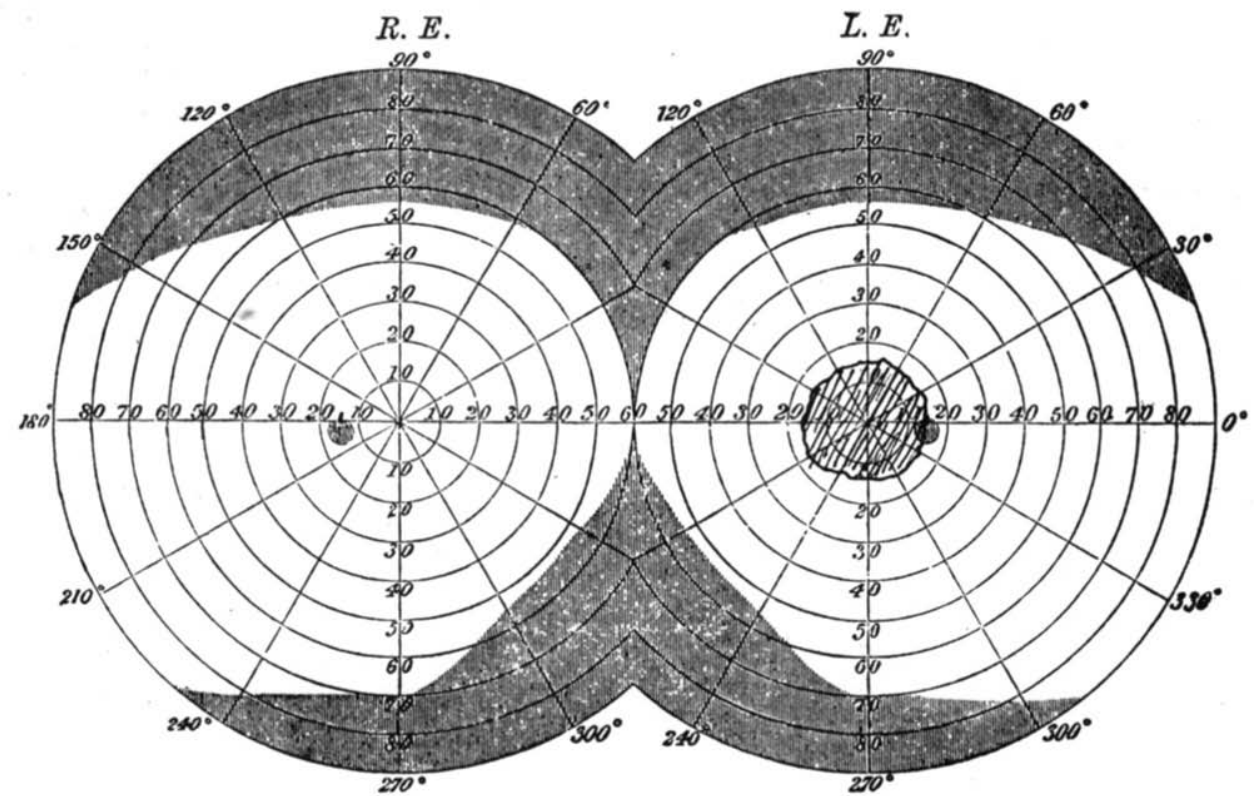

Fig. 1.-Relative central scotoma.

L. E. Case 3. January 14, 1921.

+0.37 cy. ax. $105^{\circ}$. L. V. $20 / 40$ with $+0.50 \mathrm{cy}$. ax. $90^{\circ}$. There was a small partial central scotoma in the left eye with defective color perception. Green was particularly bad, altho red was also affected. The following day the sphenoid was opened by Dr. Stuart L. Craig. No evidences of sinus disease were found. Two days later the colors were almost restored and $V .=20 / 15$. Jan. 25th, last seen, colors normal, vision $20 / 15$, scotoma entirely gone.

CASE. 4. W. Male, 67, presented himself Jan. 20, 1921, with a color scotoma in the right eye which had been pres-

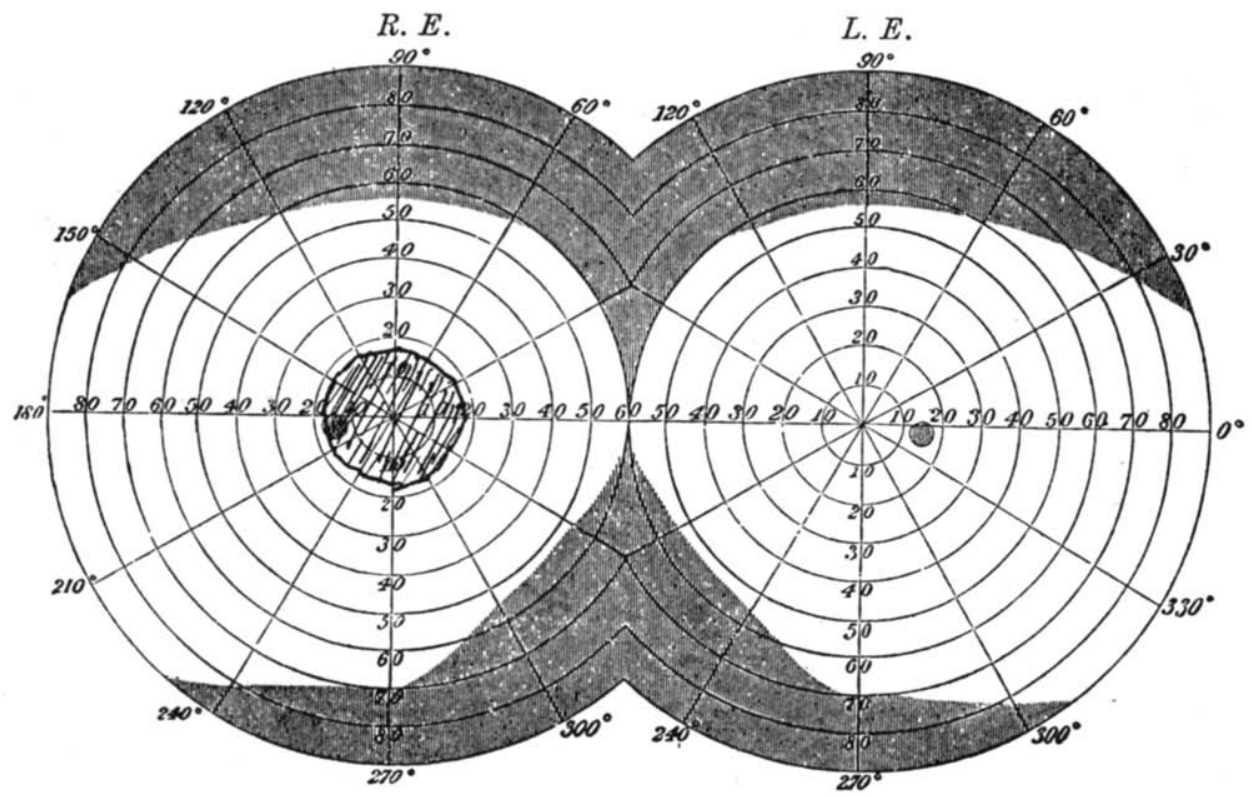

Fig. 2.-Color scotoma. R. E. Case 4. January 20, 1921. 
ent two weeks. R. V. $=20 / 50$, with his glass. L. V. $=20 / 15$, with his glass. No ophthalmoscopic findings. General health good. Several teeth with apical abscesses. Jan. 26th, the sphenoid was opened by Dr. MacKenty. No evidences of disease found. Jan. 29th, R. V. $=20 / 20-$ Jan. $31, \mathrm{R} . \mathrm{V} .=20 / 15$ - with his glass. Colors normal, scotoma gone.

CASE. 5. L. female, 18, presented herself Jan. 30, 1921. There was no perception of light in the left eye. R. V. $20 / 15$. No ophthalmoscopic findings, and general examinations negative. $\mathrm{X}$ rays showed perhaps slight increase in density near the posterior ethmoids. No direct reaction of the pupil. The conditions had been discovered a few days before, but vision had undoubtedly been failing for some days. Nasal examination negative. Feb. 7 , a radical ethmoid and sphenoid operation was done by Dr. S. McCullagh. Two days later $L$. V. $=20 / 200$. and a central color scotoma could be demonstrated. The vision has gradually improved since then until at the last observation, Feb. 28th, vision was $20 / 20$, colors normal, scotoma had disappeared.

1. Mackay. Lancet. Aug. 1908.

BIBLIOGRAPHY.

2. Brawley. Amer. Encyclo. Ophth. v. 3, p. 1811.

3. Brawley. Amer. Encyclo. Ophth. v. 3, p. 1815. 